\title{
The changing trend of head injury at Bir Hospital
}

\author{
Nilam K Khadka, M.Ch. \\ National Trauma Centre, NAMS \\ Kathmandu, Nepal. \\ Email: nilambins@gmail.com
}

Traumatic brain injury (TBI) is a critical public health and socio-economic problem throughout the world. It is a major cause of death, especially among young adults, and lifelong disability is common in those who survive. 1 The global incidence rate of TBI is estimated at 200 per 100000 people per year; however, this rate is uncertain and a likely underestimate.

Bir Hospital was established 127 years ago in 1946 B.S. and is one of the tertiary referral hospitals of Nepal. It receives many trauma victims and high proportion of them is TBI.

Now, popularly known as anti MaPaSe campaign (Zero alcohol tolerance) in motor vehicle drivers was introduced in Nepal from December 2011 AD by the traffic police of Nepal. It seems to have caused a great paradigm shift in the incidence of trauma and TBI. Comparative study was performed at Bir Hospital for two six months (similar periods of the year) in 2008 before the introduction of anti MaPaSe campaign and in 2014 after the implementation of it. The incidence of trauma, road traffic accidents (RTA), physical assault and fall were compared so were the aetiology of TBI and their prognosis. ${ }^{2}$

The total number of trauma victims was almost halved in $2014 \mathrm{AD}$ (2324) than that of $2008 \mathrm{AD}$ (4137). The number of RTA victims reduced almost by three times and physical assault victims by two times in 2014 AD than that of 2008 AD. Similarly, the TBI due to RTA reduced from $38.5 \%$ to $27 \%$ and TBI due to physical assault came down from $64 \%$ to only $12 \%$. There was not much difference in the incidences of mild and moderate head injury in those two study periods; however, there was three times reduction in the incidence of severe head injury in 2014. The most noteworthy and positive change was that the favourable outcome in terms of dichotomized Glasgow outcome scale (GOS) from TBI was 93\% in 2014 whereas it was only $74 \%$ in 2008 . The following chart clearly shows a sharp reduction in TBI mortality from 7\% in 2008 to only $4 \%$ in 2014 whereas it was grossly static for the last 35 years.

This trend of trauma and TBI victims at Bir hospital cannot be generalized to all over Nepal as we do not have
Trend of TBI mortality-Bir Hospital

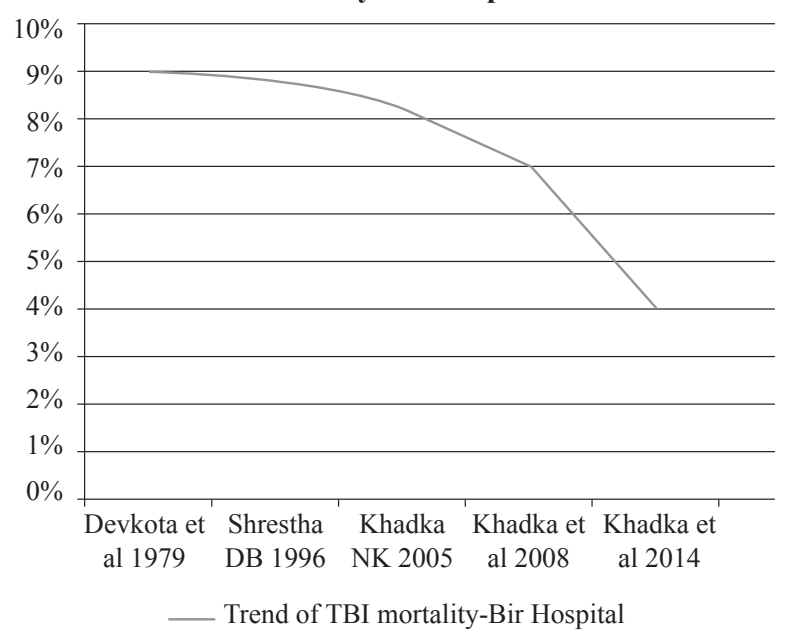

national incidence and prevalence data of TBI due to lack of national trauma registry that should be introduced immediately. However, this trend should be graciously welcomed as a healthy trend due to its good and positive impact to the society and the nation and it rightly fulfils the universal rule of prevention is better than cure. Another way of reducing the morbidity and mortality from trauma and especially TBI would be the reintroduction of compulsory use of helmet by pillion riders of the two wheelers especially motorbikes. The other strong ways are systematic introduction of pre-hospital care of trauma victims and strengthening the trauma centres in quality and quantity. These latter approaches combined with long term rehabilitation of TBI victims would complete the three pillars of a comprehensive trauma care: ResuscitationTrauma centres-Rehabilitation.

Finally, community awareness programs led by the professionalbodies like Nepalese Society of Neurosurgeons (NESON) may harbinger yet another massive strike to this modern day epidemic that is trauma and TBI.

1. Bob Roozenbeek, Andrew I. R. Maas \& David K. Menon. Changing patterns in the epidemiology of traumatic brain injury. Nature Reviews Neurology 9, 231-236 (April 2013)

2. Khadka et al. An audit of head injury at Bir hospital. Nepal Journal of Neuroscience 10:68-71, 2013 